\title{
NUMERICAL INVESTIGATIONS OF PLANAR SOLIDIFICATION OF AN UNDERCOOLED LIQUID
}

\author{
A. Esmaeeli and V. Arpaci \\ Department of Mechanical Engineering \\ and Applied Mechanics \\ The University of Michigan \\ Ann Arbor, MI 48109-2125
}

(313) 764-5242

\begin{abstract}
We investigate evolution of a planar interface during unstable solidification of a pure undercooled liquid between two parallel plates. The governing equations are solved using a front tracking/finite difference technique that allows discontinuous material properties between the phases and interfacial anisotropy. The simulations produce some of the futures of the dendritic solidification which are in good qualitative agreement with the works of the previous investigators. The effects of the physical parameters on the crystal growth and interface instability are also examined.
\end{abstract}

\section{INTRODUCTION}

Dendritic crystal growth is a long-known but poorly understood phenomenon which is central to many industrial as well as natural processes. The growth of a snowflake in the atmosphere is a familiar example of the formation of a dendritic structure in the nature. Metal casting, zone refining of semi-conductors, and protein crystallization, are just a few examples of the diverse applications of dendritic solidification.

The problem is motivated by the need to predict the crystalline microstructure of a material product during various solidification processes. Since the quality of the final product is controlled by the physical parameters such as the surface tension, the crystalline anisotropy, the initial undercooling, etc, it is quite important to understand the role of those parameters. A good review of the physics may be found in Chalmers (1964) and Langer (1980).

Compared to stable solidification, the use of numerical methods in solving the unstable solidification is relatively new. Existing numerical methods may be grouped in two classes. In one class of the methods the explicit location of the interface is not required. The phase field computations of Wheeler et al. (1993) and Kobayashi (1993), the level set computations of Sethian and Strain (1992), and the variational technique of Almgren 1993 are a few examples of the more recent works in this regard. In the other class of methods, it is required to explicitly calculate the location of interface at each time step. The computations of Smith (1981), Sullivan and Lynch (1987), Roosen and Taylor (1994), and Juric and Tryggvason (1995) are a few examples of the applications of front tracking techniques in solidification problems.

The focus of the current work is to investigate the two-dimensional interface instabilities of a solidifying undercooled melt. Our numerical technique is similar to the method developed by Juric and Tryggvason (1995). 


\section{NUMERICAL METHOD}

Consider a domain consisting of a liquid and its solid phase. The density of the liquid and solid are assumed to be the same and equal, but all the other material properties are taken to be different but constant within each phase. The following equations needs to be satisfied inside each phase

$$
\frac{\partial c T}{\partial t}=\nabla \cdot(k \nabla T)
$$

and across the front

$$
\begin{gathered}
{[[h]]_{s}^{l} V=k\left[[\nabla T]_{s}^{l} \cdot \bar{n},\right.} \\
T_{f}=T_{e q}-\frac{\sigma(\bar{n}) \kappa T_{e q}}{L}-\nu(\bar{n}) V .
\end{gathered}
$$

The first equation is simply the unsteady heat conduction equation in conservative form. Here, $T$ is the temperature, $c$ is the heat capacity, and $k$ is the heat conductivity. The second equation is the statement of energy jump condition across the interface. $[[h]]_{s}^{l}$ is the difference between the liquid and solid enthalpies at the front temperature, and $\bar{n}$ is the unit normal vector at the front. Equation (3) is the Gibbs-Thomson relation which needs to be satisfied across the front. In this equation, $T_{f}$ is the interface temperature, $T_{e q}$ is the fusion temperature (which is used also as a reference temperature), $\sigma(\bar{n})$ is the anisotropic surface tension, $\kappa$ is the front curvature, $L$ is the latent heat of fusion, $\nu(\bar{n})$ is the kinetic mobility coefficient, and $\mathrm{V}$ is the front velocity. [[h] $]_{s}^{l}$ is defined through the following thermodynamic relation:

$$
[[h]]_{s}^{l} V=L+\left(c_{l}-c_{s}\right)\left(T_{f}-T_{e q}\right)
$$

Rather than writing the energy equation separately for each of the phases and coupling the equations through the energy jump condition, a formulation is used which is valid for the entire domain and takes the energy jump condition into account:

$$
\frac{\partial c T}{\partial t}=\nabla \cdot(k \nabla T)+\oint \bar{h} V \delta\left(\bar{x}-\bar{x}^{f}\right) d s,
$$

where, $\bar{h}$ is defined to be:

$$
\bar{h}=L+\left(c_{s}-c_{l}\right) T_{e q} .
$$

Away from the interface (where delta function vanishes), the above formulation gives the original energy equation and integration of the above equation over a shrinking area which contains the interface results in the energy jump condition.

The computations start with constructing the initial temperature field $\left(T^{n}\right)$ and the material property fields $\left(c^{n}, k^{n}\right)$ using the known position of the front at $t=0$, where $n=1$. The velocities of the front points at the next time step $\left(V^{n+1}\right)$ are guessed and the front is moved to a new position. The heat conductivity field is constructed at the new position $\left(k^{n+1}\right)$, the front temperature is interpolated from the grid temperature, the source term in the right hand side of equation (5) is distributed to the grid points, and equation (5) is solved for $T^{n+1}$. Next, the front temperature is computed using grid temperature $T^{n+1}$ and equation (3) is checked for the front points. If this equation is satisfied for all the front points within a predetermined tolerance, the heat capacity field is then constructed $c^{n+1}$ and the computations proceed to the next time step. Otherwise, a new guess is made for the velocities of the front points and the above procedure is repeated. 
The results depend on $L, c_{l}, c_{s}, k_{l}, k_{s}, \sigma, T_{\infty}-T_{e q}, \nu$, and a suitable length scale, $l$. Nondimensionalization of the above parameters leads to the Stefan number; $S t=c_{s}\left(T_{\infty}-T_{e q}\right) / L$; the capillary number; $C a=c_{s} T_{e q} \sigma / L^{2} l ; \Pi=\nu k_{s} / L l$, and the ratios of material properties; $\lambda=k_{l} / k_{s}$, and $\gamma=c_{l} / c_{s}$.

\section{RESULTS}

We start our analysis by considering the evolution of a solid-liquid interface during the planar solidification of an undercooled liquid in a two-dimensional domain. The domain is confined by insulated walls at the top and the bottom and is periodic in the horizontal direction. Initially, the upper portion of the domain is filled with liquid at $T_{\infty}$ which overlays on a thin layer of solid at $T_{e q}$. The interface between the liquid and the solid is perturbed slightly to trigger the instability. For all the simulations in this paper, the domain size is $1 \times 2$ and it is resolved by a $150 \times 300$ grid. The interface shape is described by:

$$
h=h_{c}+\epsilon \cos \left(2 \pi N x / x_{l}\right)
$$

where, $h_{c}, \epsilon, N$, and $x_{l}$ are the interface average height, perturbation amplitude, perturbation mode, and domain width in $x$ direction.

The first frame of figure (1) shows the initial position of the interface for one of our simulations. Here, $h_{c}=0.1, \epsilon=-0.01$, and $N=4$. The subsequent frames shows the evolution of the interface at equispaced times. The nondimensional numbers for this run are $S t=-0.8, C a=0.008$, $\Pi=0.004, \lambda=1$, and $\gamma=1$. As the interface is set to motion, due to the initial temperature gradients, it is seen that the interfacial perturbations start to grow (frames 2-4). The figure shows the emergence of fingers with the length scales of the same order as the domain size. The growth of the interface instability is due to the combined effect of the temperature gradient, the surface tension energy, and the kinetic mobility coefficient at the interface. Since the liquid is undercooled, it is possible for the interface shape to become unstable and develop a small scale structure. The surface tension acts to stabilize the interface against short-wavelength perturbations. Since the temperature field in the solid phase is almost uniform, the velocity of the interface is proportional to the temperature gradient at the liquid side of the interface. Therefore, the interface moves faster in the regions of positive curvature and slower in the regions of negative curvature. In the positive curvature regions, the surface tension acts to reduce the interface temperature at the tip of the protrusion below $T_{e q}$. This flattens the temperature profile and thus reduces the front velocity. Similar argument goes for the negative curvature regions where the surface tension effect is to raise the interface temperature above $T_{e q}$.

The left frame of the figure (2) compares the effect of heat conductivity ratio for simulations at $\lambda=0.5$ (bottom curve), $\lambda=1$ (middle curve), and $\lambda=2.0$ (top curve) at $t=0.06$. It is seen that heat conductivity ratio only affects the growth rate and it has no effect on the interface instability. The higher the heat conductivity ratio, the higher the growth rate. The increase in this ratio results a higher heat transfer rate in the liquid at the interface. This in turn results in a higher front velocity (equation 2).

The middle frame shows the results of three simulations at $\gamma=0.5$ (bottom curve), $\gamma=1$ (middle curve), and $\gamma=1.2$ (top curve) at $t=0.025$. It is seen that the increase in the liquid to solid heat capacity ratio increases the growth rate and the interface instability. The increase 

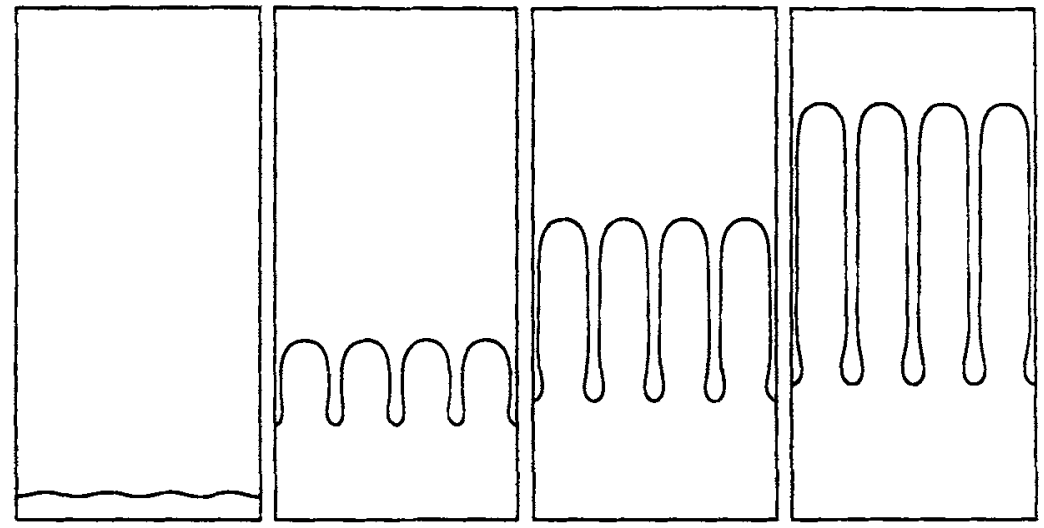

Figure 1: Solidification of an Underooled Liquid. The nondimensional numbers for this run are $S t=-0.8, C a=0.008, \Pi=0.004, \lambda=1$, and $\gamma=1$. Times are $0.0,0.03,0.06$, and 0.09 . The Resolution is $150 \times 300$ in a $1 \times 2$ box.

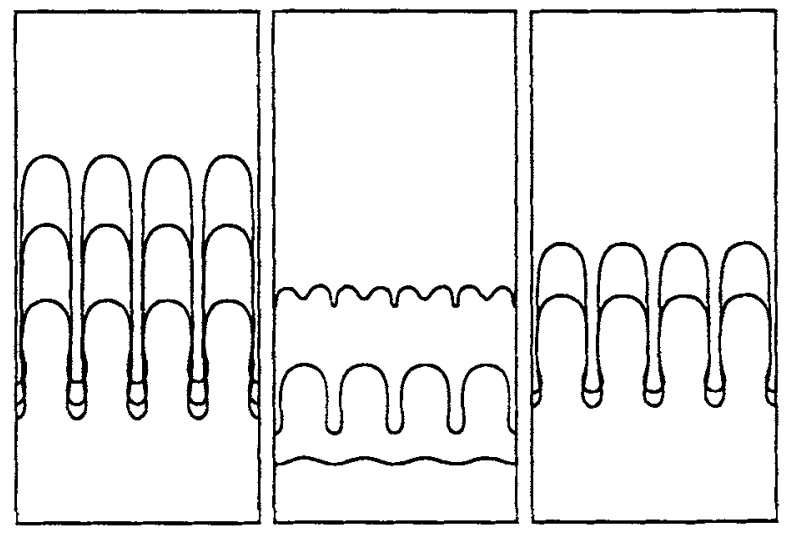

Figure 2: Effect of Heat Conductivity Ratio $(t=0.06$, left frame), Heat Capacity Ratio $(t=0.025$, middle frame), and Surface Tension Anisotropy ( $t=0.055$, right frame).

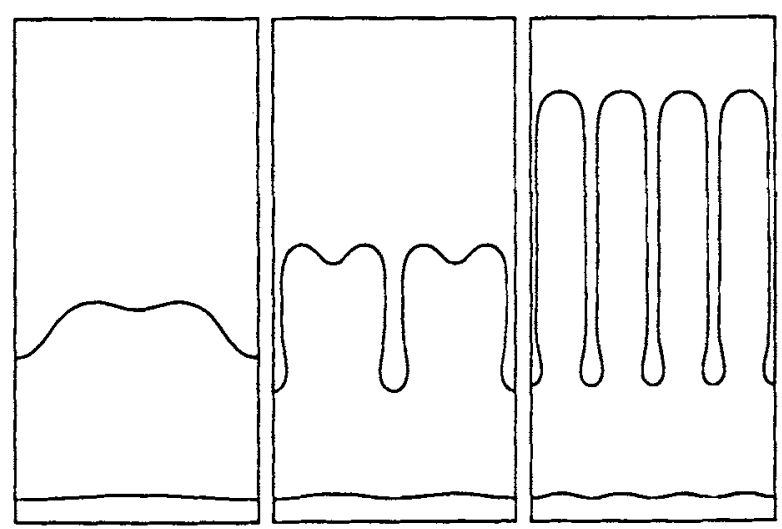

Figure 3: Effect of Wave Number of Initial Perturbation $(t=0.095)$. Here, $N=1$ (left frame), $N=2$ (middle frame), and $N=4$ (right frame). 
in growth rate is the result of a higher temperature gradient at the liquid side of the interface. Since a material with a higher heat capacity will have a higher resistance to thermal adjustments, therefore, it has a lower ability to diffuse the latent heat released at a given time. As a result, the temperature gradient in the liquid will be higher. This in turn results in a higher interface velocity. To investigate the increase in interface instability, we first eliminate $\left(T_{f}-T_{e q}\right)$ from equation 4 by using equation 3 and then substitute the resulting equation in the equation 2 . It is seen that for $\gamma>1$, the front velocity increases in the regions with positive curvature and decreases in the negative curvature regions. Consequently, the interface becomes more unstable as we increase the heat capacity ratio. On the other hand, for $\gamma<1$, the interface velocity decreases in the positive curvature regions and increases in the negative curvature regions. As a result, the front evolves toward a more stable shape. Indeed, continuation of the run with $\gamma<1$ showed that eventually the initial perturbation was dumped out.

In the previous computations, the surface tension and the kinetic mobility coefficient was constant. This is not true generally. In the real life applications, however, the surface tension may be anisotropic. When the surface tension is anisotropic, the growth will be enforced in the directions that are favored by the anisotropy. The right frame compares the effect of anisotropy in the surface tension for two simulations at $t=0.055$. Here, $\sigma(\bar{n})=\sigma_{0}$ for the top curve and $\sigma(\bar{n})=\sigma_{0}(1-0.5 \cos (2 \theta))$ for the bottom curve. As is expected, the growth rate is higher for the interface with isotropic surface tension. Moreover, it is seen that the corresponding fingers in the two curves have different curvatures as a result of unequal surface tensions.

Next we investigate the effect of wavelength of the initial perturbation. Figure (3) compares the results of simulations at $N=1$ (left frame), $N=2$ (middle frame), and $N=4$ (right frame) at $t=0.095$. The bottom curve in each of the frames is the initial position of the interface. While the interface with the shortest wavenumber has the fastest growth rate, the other two interfaces are more unstable since they start to bifurcate. The previous instability analyses have shown the existence of a cut off wave number, $k=k_{c}$, below which the interface is stable (see for example Langer, 1980). Moreover, the growth rate does not change monotonically with the wave number and there exists a critical wavenumber, $k=k_{\max }$, which results in the maximum growth rate. We have not compared our results with the existing theories in any quantitative way, but we intend to do so in the near future.

\section{Conclusions}

Numerical simulations of unstable planer solidification is presented. The results show the effect of the material properties ratios, anisotropy, and wavelength of the initial perturbation on the interface growth and instability. It was shown that while the change in heat conductivity ratio only affects the growth rate, a small change in heat capacity ratio has a great impact on both the growth rate and the interface instability.

\section{Acknowledgments}

The computations were done on the computers at Laboratory for Scientific Computing (LaSc) of the University of Michigan. We would like to thank Professor James Holloway for his help using LaSc facilities. 


\section{References}

Almgren, R. (1993) "Variational Algorithm and Pattern Formation in Dendritic Solidification," J. Comput. Phys., 106: 337-354.

Chalmers, B. (1964) Principles of Solidification, Wiley, New York.

Juric, D. and G. Tryggvason (1996) "A Front-Tracking Method for Dendritic Solidification," J. Comput. Phys., 123: 127-148.

Kobayashi, R. (1993) "Modeling and Numerical Simulations of Dendritic Crystal Growth," Physica D, 63: 410-413.

Langer, J. S. (1980) Rev. Mod. Phys., 52.

Roosen, A. and J. Taylor (1964) "Modeling of Crystal Growth in a Diffusion Field using Fully Faceted Interfaces," J. Comput. Phys., 114: 113-128.

Sethian, J. A. and J. Strain (1992) "Crystal Growth and Dendritic Solidification," J. Comput. Phys., 98: 231-253.

Smith, J. B. (1981) "Shape Instabilities and Pattern Formation in Solidification: A New Method for Numerical Solution of the Moving Boundary Problem," J. Comput. Phys., 39: 112-127.

Sullivan, J. M. and D. R. Lynch (1987) "Finite Element Simulation of Planar Instabilities during Solidification of an Undercooled Melt," J. Comput. Phys. 69:81-111.

Wheeler, A. A., B. T. Murray, and R. J. Schaefer (1993) "Computations of Dendrites using a Phase Field Model," Physica D, 66: 243-262. 